\title{
Dipeptidyl peptidase-4 inhibition with linagliptin prevents western diet-induced vascular abnormalities in female mice
}

\author{
Camila Manrique ${ }^{1,2,4 \dagger}$, Javad Habibi 1,2,4 , Annayya R. Aroor ${ }^{1,2,4}$, James R. Sowers ${ }^{1,2,3,4,5}$, Guanghong Jia 1,2,4, \\ Melvin R. Hayden 1,2, Mona Garro 1,2,4, Luis A. Martinez-Lemus ${ }^{3,5}$, Francisco I. Ramirez-Perez ${ }^{3,5}$, Thomas Klein ${ }^{6}$, \\ Gerald A. Meininger ${ }^{3,5}$ and Vincent G. DeMarco $1,2,3,45^{*}$
}

\begin{abstract}
Background: Vascular stiffening, a risk factor for cardiovascular disease, is accelerated, particularly in women with obesity and type 2 diabetes. Preclinical evidence suggests that dipeptidylpeptidase-4 (DPP-4) inhibitors may have cardiovascular benefits independent of glycemic lowering effects. Recent studies show that consumption of a western diet (WD) high in fat and simple sugars induces aortic stiffening in female C57BL/6J mice in advance of increasing blood pressure. The aims of this study were to determine whether administration of the DPP-4 inhibitor, linagliptin $(L G T)$, prevents the development of aortic and endothelial stiffness induced by a WD in female mice.

Methods: C56Bl6/J female mice were fed a WD for 4 months. Aortic stiffness and ex vivo endothelial stiffness were evaluated by Doppler pulse wave velocity (PWV) and atomic force microscopy (AFM), respectively. In addition, we examined aortic vasomotor responses and remodeling markers via immunohistochemistry. Results were analyzed via 2-way ANOVA, $p<0.05$ was considered as statistically significant.

Results: Compared to mice fed a control diet (CD), WD-fed mice exhibited a $24 \%$ increase in aortic PWV, a five-fold increase in aortic endothelial stiffness, and impaired endothelium-dependent vasodilation. In aorta, these findings were accompanied by medial wall thickening, adventitial fibrosis, increased fibroblast growth factor 23 (FGF-23), decreased Klotho, enhanced oxidative stress, and endothelial cell ultrastructural changes, all of which were prevented with administration of LGT.

Conclusions: The present findings support the notion that DPP-4 plays a role in development of WD-induced aortic stiffening, vascular oxidative stress, endothelial dysfunction, and vascular remodeling. Whether, DPP-4 inhibition could be a therapeutic tool used to prevent the development of aortic stiffening and the associated cardiovascular complications in obese and diabetic females remains to be elucidated.
\end{abstract}

Keywords: Pulse wave velocity, Atomic force microscopy

\section{Background}

Increased arterial stiffness is a cardiovascular (CV) biomarker strongly associated with hypertension, diastolic heart failure, chronic kidney disease and stroke $[1,2]$.

\footnotetext{
${ }^{*}$ Correspondence: demarcov@missouri.edu

${ }^{\dagger}$ Camila Manrique and Javad Habibi contributed equally to this work

1 Department of Internal Medicine, Division of Endocrinology, Diabetes and Metabolism, University of Missouri-Columbia School of Medicine, One Hospital Drive, Columbia, MO 65212, USA

Full list of author information is available at the end of the article
}

Importantly, obese, insulin resistant and diabetic women develop vascular stiffness more frequently than men and this circumstance might account for the disproportionate incidence of cardiovascular disease (CVD) in insulin resistant women $[3,4]$. The widespread consumption of diets high in refined carbohydrates and fat [western diet (WD)] is one of the driving forces behind the alarming growth in the incidence of obesity and insulin resistance $[5,6]$. The progression to CVD in the setting of obesity and type 2 diabetes is likely initiated by endothelial 
dysfunction and several associated vascular abnormalities, including altered vascular tone, extracellular matrix remodeling and adventitial dysfunction, all of which could promote arterial stiffness $[7,8]$. We recently tested this notion and reported that after 4 months of WD feeding, the aorta of normotensive female C57BL/6J mice exhibits altered nitric oxide (NO)-dependent vasodilation associated with aortic and endothelial stiffness, increased aortic wall thickening and fibrosis, oxidative stress and inflammation [9]. Recent reports from our laboratory indicate that therapies targeting vascular stiffness could potentially improve CV outcomes in insulin resistance models $[7,9]$. In this regard, the use of dipeptidyl peptidase-4 (DPP-4) inhibitors may be of interest. DPP-4 is an exopeptidase that circulates in plasma and is also expressed on the surface of multiple cell types including endothelial and vascular smooth muscle cells, as well as immune cells [10]. Although DPP-4 inhibitors were developed to control hyperglycemia, it is now established that these inhibitors have effects beyond glycemic regulation [11]. The rationale for using DPP-4 inhibitors to target vascular stiffness relates to the potential of these compounds to improve endothelial function [11, 12]. Even though the beneficial effects of DPP-4 inhibition on the cardiovascular system have been explored in both animal models and in type 2 diabetic patients [11], to the best of our knowledge, the potential role of these compounds to target vascular stiffness in a female rodent model of insulin resistance due to over-nutrition without frank hyperglycemia or blood pressure elevation has not been studied. In the present investigation, we tested whether administering linagliptin (LGT), a long acting and specific DPP-4 inhibitor, to female C57BL/6 J mice fed a WD for 4 months, could ameliorate the development of WD-induced aortic and endothelial cell (EC) stiffness. Herein, we report that DPP-4 inhibition prevents the development of WD-induced aortic and EC stiffness in overweight female mice. The mechanism, in part, appears a consequence of preventing abnormalities in aortic endothelium-dependent vasorelaxation, oxidative stress, medial wall thickening and fibrosis, as well as expression of FGF-23 and Klotho.

\section{Methods}

\section{Animal models}

Three week old C57BL/6 J female mice were purchased from Jackson Laboratories (Bar Harbor, ME) and cared for in accordance with National Institutes of Health guidelines. All procedures were approved in advance by the Institutional Animal Care and Use Committee of the University of Missouri. Beginning at 4 weeks of age, mice were fed a WD consisting of high fat (46\%) and high carbohydrate as sucrose $(17.5 \%)$ and high fructose corn syrup (17.5 \%) for 4 months (Test Diet $58 \mathrm{Y} 1$ with high refined carbohydrate and high fat, 5APC, Richmond, Indiana). Parallel groups of age-matched control mice were fed a modified control diet $(C D)$ for the same period of time (Test Diet 58Y2). Mice were provided water ad libitum while housed in groups of four under a 12-hour/day illumination regimen. Based on a daily food intake of $0.1 \mathrm{~g}$ of chow per $\mathrm{g}$ body weight observed in a previous cohort of WD-fed mice, we added LGT (BI 1356; (R)-8-(3-aminopiperidin-1-yl)-7-but-2-ynyl-3-methyl-1-(4-methyl-quinazolin2-ylmethyl)-3,7-dihydropurine-2,6-dione) [13] to mouse chow so that the final concentration in chow was $83 \mathrm{mg} \mathrm{LGT} \mathrm{kg} \mathrm{kg}^{-1}$ to achieve a dose and plasma level of approximately $8 \mathrm{mg} \mathrm{kg}^{-1} \mathrm{day}^{-1}$ and approximately $50-100 \mathrm{nM}$, respectively [14]. Four groups of mice were utilized and they include mice fed a control diet without LGT (CDC), control diet with LGT (CDL), western diet without LGT (WDC) and western diet with LGT (WDL). Different sub-cohorts underwent the procedures described below.

\section{Body weight}

To assess weight gained during the 4 month experiment, mice were weighed immediately prior to the start of the experiment at 4 weeks of age and before sacrifice.

\section{DPP-4 activity assay}

DPP-4 activity assay was performed as previously described [15]. Briefly, blood was collected at the time of killing in EDTA tubes and plasma was stored at $-80{ }^{\circ} \mathrm{C}$. $20 \mu \mathrm{L}$ plasma was diluted in DPP-4 assay buffer and substrate, 200 M H-Ala-Pro-AFC (I-1680; Bachem), was added. Fluorescence was measured with a Synergy Microplate Reader at excitation wavelength of $405 \mathrm{~nm}$ and emission wavelength of $535 \mathrm{~nm}$ [15].

\section{Aortic stiffness by in vivo pulse wave velocity (PWV)}

Doppler ultrasound (Indus Mouse Doppler System, Webster, TX) was performed under isoflurane anesthesia, according to a previously established protocol [9], to evaluate PWV for in vivo determination of arterial stiffness. PWV is affected by age, blood pressure, and heart rate. Consequently, PWV is reported both in $\mathrm{m} / \mathrm{s}$ (unadjusted), as well as normalized to heart rate (HR). Limiting the WD feeding period to 4 months allowed us to avoid any effects of increased blood pressure on vascular stiffening as 4 months of WD does not induce an elevation of blood pressure in female C57BL/6 mice [9].

\section{Ex vivo vasomotor responses in the aorta}

Aortic vasomotor responses were examined as previously described [9]. Briefly, vessels were preconstricted with $\mathrm{U} 46619(100 \mathrm{nM})$ and responses to acetylcholine (ACh) 
(1 $\mathrm{nm}$ to $100 \mu \mathrm{mM}$ ), and to insulin (INS: Novolin R, Novo Nordisk; $0.1-300 \mathrm{ng} / \mathrm{mL}$ ) were assessed by cumulative addition of agonist to an isolated vessel bath as previously described [9]. At the end of each experiment, the PSS bath solution was replaced with $\mathrm{Ca}^{2+}$-free PSS to determine maximal relaxation.

\section{Atomic force microscopy (AFM) for force measurement and stiffness calculation}

AFM was used to evaluate stiffness of ECs in enface aortic preparations of the thoracic aorta as previously described [9]. Stiffness (elastic modulus) of the EC surface was measured by AFM using a nano-indentation protocol [9].

\section{Aortic remodeling and protein quantification}

A segment of thoracic aorta was fixed in $3 \%$ paraformaldehyde, dehydrated, paraffin embedded, and transversely sectioned in $5 \mu \mathrm{m}$ slices. To evaluate peri-aortic fibrosis, as previously described, the sections were stained with picro sirius red. To evaluate the medial thickness the sections were stained with Verhoeff-von Gieson [9]. The pink color intensities and areas were quantified as "average gray scale intensities" in five images per mouse with MetaVue software. Aortic oxidative stress was assessed by quantifying brown color in the endothelium, media and adventitia of the aorta which is indicative of 3-nitrotyrosine formation (1:150 dilution; Millipore, Billerica, MA), as previously described [16]. The expression level of fibroblast growth factor-23 (FGF-23) (1:50 dilution; Novus NBP1-55830), klotho (1:100 dilution; Novus NBP1-76511) and advanced glycation endproducts (AGE) (1:100 dilution; Abcam ab23722) was determined by quantifying of signal intensities on the fluorescent images on the different components of aorta as "average gray scale intensities".

\section{Transmission electron microscopy (TEM)}

TEM was performed as previously described using a JEOL 1400-EX TEM to determine ultrastructural changes associated with increased vascular stiffness [17]. Briefly, aortic samples were collected and fixed in $2 \%$ glutaraldehyde $/ 2 \%$ paraformaldehyde followed by secondary fixation with $1 \%$ osmium tetroxide, embedded in Epon-Spurr's resin, sectioned at $85 \mathrm{~nm}$, and stained with uranyl acetate/Sato's triple lead stain.

\section{Statistical analysis}

Results are reported as the mean \pm SE. Statistical analysis was primarily by two-way ANOVA followed by post hoc $t$ tests (Bonferroni) to examine differences in outcomes between mice fed CD or WD and administered LGT in mouse chow or with no LGT in the chow (Sigma
Plot 13.0, Systat Software). Aortic dilator responses are presented as percent maximal relaxation, calculated as $[(\mathrm{Fb}-\mathrm{Fd}) /(\mathrm{Fb}-\mathrm{Fmin})]^{*} 100$, where $\mathrm{Fd}$ is force after a drug intervention, $\mathrm{Fb}$ is baseline force, and Fmin is minimal force obtained during passive conditions.

\section{Results}

Body weight, plasma DPP-4 activity and AGE

Body weights of 20-week old WDC and WDL mice were of similar weights and heavier compared to those of their respective lean counterparts (Additional file 1: Table S1; Fig. 1a). Percent body weight gain during the 4 month study period was $71 \pm 4$ and $93 \pm 6 \%$ for CDC and WDC ( $p>0.05)$, respectively, and $74 \pm 7 \%$ and $88 \pm 7 \%$ for CDL and WDL ( $\mathrm{p}>0.05$ ), respectively. Thus, DPP-4 inhibition had no significant effect on body weight (Additional file 1: Table S1; Fig. 1a) within each dietary regime during the test period. As expected, DPP-4 plasma activity was significantly decreased in both groups treated with LGT without a diet effect (Fig. 1b). As AGE have been associated with vascular stiffness in some models of insulin resistance $[18,19]$, we examined the presence of AGE in the aortic wall (Fig. 1c). WD significantly increased the accumulation of AGE in the vascular wall, and this was not affected by administration of LGT.

\section{DPP-4 inhibition prevented WD-induced aortic and EC stiffness \\ In vivo}

PWV was determined in mice following four months on CD or WD (Fig. 2a). PWV did not vary significantly in CDC mice (Fig. 2a; Table 1). PWV was elevated in the WDC group compared to CDC ( $<<0.001)$ and administration of LGT prevented the WD-induced increase in PWV ( $\mathrm{p}<0.001)$ (Fig. 2a; Table 1). No differences in PWV were observed between CDL and WDL groups $(\mathrm{p}=0.363)$. Normalization of PWV to HR yielded similar results as shown in the adjusted PWV analysis (Table 1).

\section{Ex vivo}

We recently reported increased EC stiffness in aortic explants utilizing AFM after 4 months of WD in female mice [9]. Similarly, in this investigation we observed increased surface stiffness in the EC from WD-fed mice ( $\mathrm{p}<0.05 \mathrm{CDC}$ vs. WDC) and this finding was prevented by LGT administration ( $\mathrm{p}<0.05$ for WDC vs. WDL and $\mathrm{p}>0.05$ for CDC vs. WDL) (Fig. 2b).

\section{DPP-4 inhibition improves endothelial dependent responses in the aorta}

We recently reported that 4 months of WD feeding in female $\mathrm{C} 57 \mathrm{BL} / 6 \mathrm{~J}$ mice induced impaired protein kinase B (Akt)/endothelial nitric oxide synthase (eNOS) 

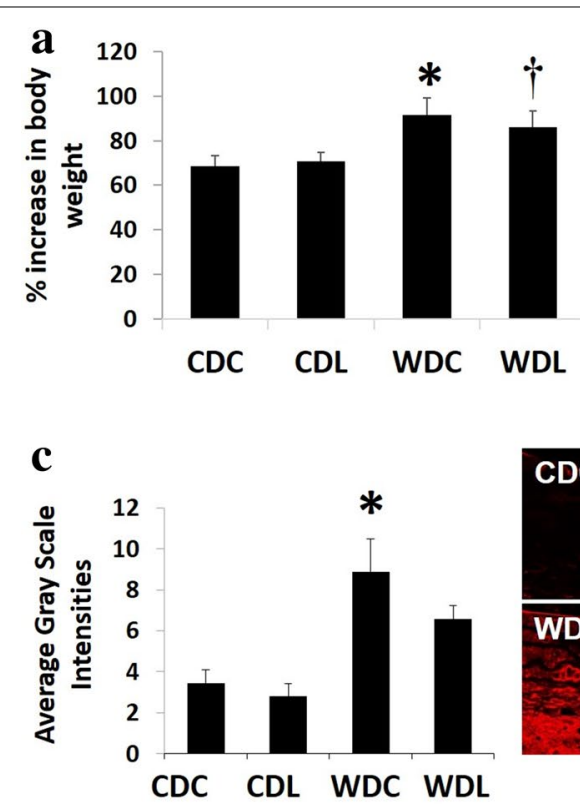
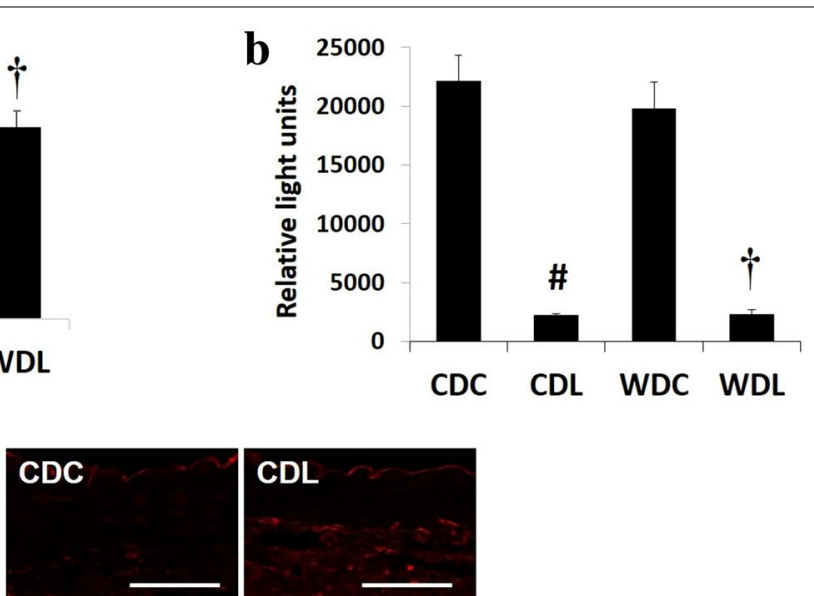

CDL

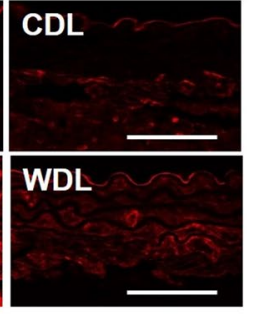

Fig. 1 Linagliptin (LGT) has neutral effects on body weight and aortic advanced glycation end-products (AGE). a WD feeding for 4 months resulted in significant weight gain in both the cohorts. b Plasma DPP-4 activity was significantly decreased with LGT treatment. c AGE immunostaining in aorta was significantly increased in WDC. LGT treatment did not decrease it significantly. Quantification and representative images shown. Values are mean \pm SE. CDC control diet control, CDL control diet linagliptin, WDC western diet control, WDL western diet linagliptin. Post-hoc comparisons within a time point; ${ }^{*} p<0.05$ CDC vs WDC; ${ }^{*} p<0.05$ CDC vs CDL; ${ }^{\dagger} p<0.05$ WDC vs WDL. Scale bars represent 50 mu
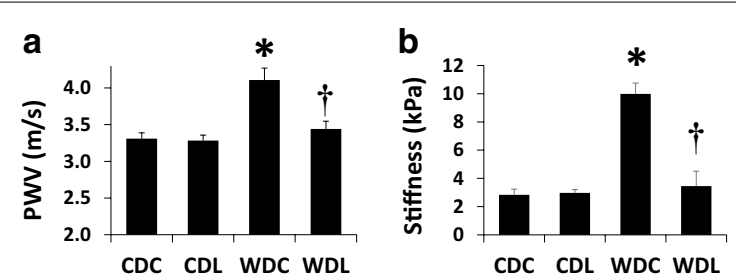

Fig. 2 LGT prevents development of in vivo aortic stiffening, as well as endothelial stiffening in ex vivo aortic explants. a Pulse wave velocity (PWV) measured after 4 months on experimental diets. b Force measurements were acquired by interaction between a cantilever tip and the EC surface of aortic explants from mice after 4 months on WD. Values are mean \pm SE. CDC control diet control, CDL control diet linagliptin, WDC western diet control and WDL western diet linagliptin. Post-hoc comparisons within a time point; ${ }^{*} p<0.05$ CDC vs WDC; ${ }^{\dagger} \mathrm{p}<0.05$ WDC vs WDL

signaling and this was associated with impaired aortic endothelium-dependent vasodilation [9]. Herein, we evaluate Akt/eNOS signaling in aortic rings by functional assay to examine the vasodilatory responses to $\mathrm{ACh}$ and insulin. Four months of WD feeding resulted in impaired aortic endothelium-dependent vasodilatory responses to $\mathrm{ACh}$ and insulin in the WDC compared to CDC (Fig. 3a, b). These defects were prevented in the WDL group (Fig. 3a, b).

\section{DPP-4 inhibition prevents vascular remodeling}

We have previously shown that WD feeding results in increased aortic remodeling. Similarly, in the present investigation, aortic medial thickness and fibrosis were significantly increased with WD feeding compared to CDC (Fig. 4a-d). Importantly, aortic fibrosis and increased medial thickness were prevented with DPP-4 inhibition (Fig. 4a-d).

\section{DPP-4 inhibition prevents the increase in WD-induced ultrastructure changes in EC}

Increased activation of the contractile apparatus of EC has been associated with the genesis of vascular stiffness [20]. In this study, we examined the ultrastructural characteristics of the aorta in the different experimental groups (Fig. 5a-d). WD was associated with loss of luminal endothelial cytoplasmic elongation and nuclear contraction and these abnormalities were prevented in the WDL group (Fig. 5c, d).

\section{DPP-4 inhibition prevents oxidative stress}

We have previously shown that vascular stiffness induced by WD feeding in females was associated with increased vascular oxidative stress [9]. Here in we showed that WD increased aortic oxidative stress assessed by the presence of 3-nitrotyrosine immunostaining (Fig. 6). Importantly, 
Table 1 Aortic stiffness in untreated western diet (WD)-fed mice compared to untreated control diet (CD) fed mice as indicated by determinations of aortic pulse wave velocity (PWV)

\begin{tabular}{|c|c|c|c|c|c|c|}
\hline Pulse wave velocity & Main effect & p value & $\mathrm{CDC}[6]$ & CDL [8] & WDC [10] & WDL [10] \\
\hline \multirow[t]{3}{*}{4 months PWV $\left(\mathrm{m} \mathrm{s}^{-1}\right)$} & Diet & 0.001 & $3.31 \pm 0.08$ & $3.28 \pm 0.07$ & $4.11^{* \dagger} \pm 0.16$ & $3.44 \pm 0.11$ \\
\hline & Treatment & 0.001 & & & & \\
\hline & Interaction & 0.017 & & & & \\
\hline \multirow[t]{3}{*}{ Heart rate (bpm) } & Diet & 0.036 & $442 \pm 22$ & $439 \pm 26$ & $397^{*} \pm 12$ & $415 \pm 5$ \\
\hline & Treatment & 0.639 & & & & \\
\hline & Interaction & 0.496 & & & & \\
\hline \multirow[t]{3}{*}{4 months PWV $\left(\mathrm{m} \mathrm{s}^{-1} \mathrm{HR}^{-1} 100\right)$} & Diet & 0.006 & $0.76 \pm 0.04$ & $0.77 \pm 0.05$ & $1.05^{* \dagger} \pm 0.06$ & $0.83 \pm 0.03$ \\
\hline & Treatment & 0.091 & & & & \\
\hline & Interaction & 0.072 & & & & \\
\hline
\end{tabular}

Also shown, is heart rate (HR) and PWV normalized to HR. Administration of linagliptin (WDL) prevented the increase in PWV observed WD-fed mice (WDC). Sample sizes are noted in parentheses. Values are mean $\pm \mathrm{SE}$

$C D C$ control diet control, $C D L$ control diet linagliptin, WDC western diet control and WDL western diet linagliptin. Post-hoc comparisons

${ }^{*} \mathrm{p}<0.05$ CDC vs WDC; ${ }^{\dagger} \mathrm{p}<0.05$ WDC vs WDL
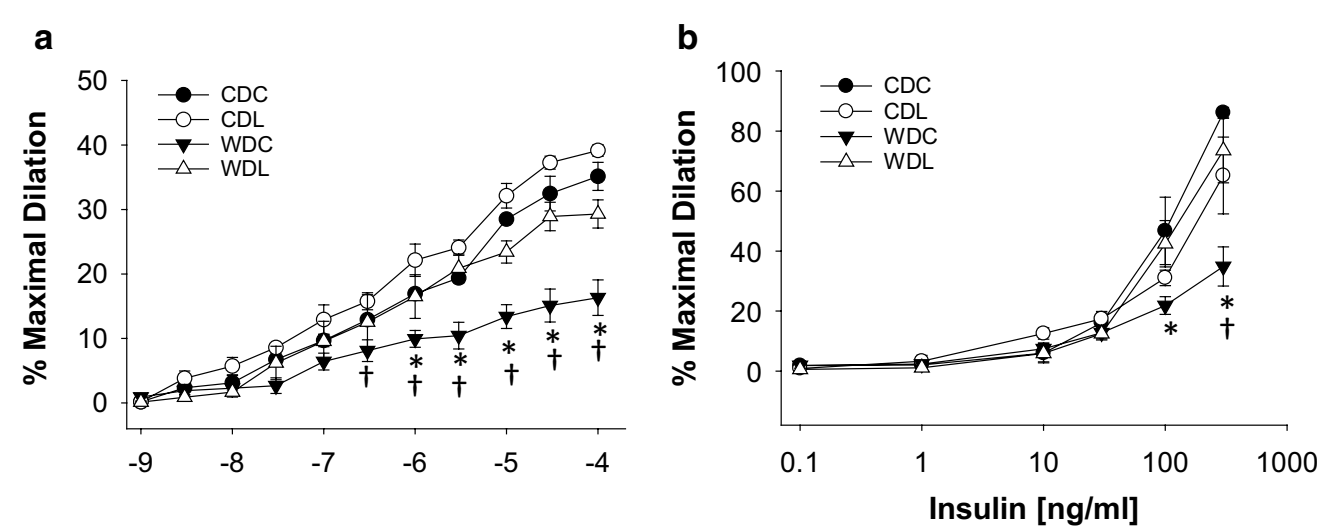

Fig. 3 Vasodilator responses of isolated aortic rings to the endothelium-dependent dilators acetylcholine (a) and insulin (b). Values are mean \pm SE. $C D C$ control diet control, $C D L$ control diet linagliptin, WDC western diet control and WDL western diet linagliptin. Post-hoc comparisons within a time point; ${ }^{*} p<0.05$ CDC vs WDC; ${ }^{\dagger} p<0.05$ WDC vs WDL

DPP-4 Inhibition ameliorated aortic oxidative stress by normalization of 3-nitrotyrosine induced by WD feeding (Fig. 6).

DPP-4 inhibition prevents WD-induced changes in FGF-23 and Klotho expression in the vascular wall

FGF-23 is associated with arterial stiffness and increased risk of CVD [21-23]. We have recently shown that this WD feeding paradigm results in increased FGF23 in the vascular wall [24]. Furthermore, decreased Klotho expression, another protein involved in vascular calcification, is known to exacerbate diet-induced vascular stiffness [9]. Herein we examined whether WD induces changes in FGF-23 and Klotho expression in the aorta, and if so, whether DPP-4 inhibition prevented that change (Fig. 7). As anticipated, we observed a significant increase in FGF-23 in the aortic endothelium and adventitia of the WDC group compared with CDC, and this increase was prevented in the WDL group (Fig. $7 \mathrm{a}-$ c). In addition, Klotho expression was significantly decreased by WD feeding and DPP-4 inhibition prevented this deficiency in the vascular wall (Fig. $7 d-g$ ).

\section{Discussion}

Collectively, the results of this investigation support the hypothesis that DPP-4 activation plays an important role in the development of aortic stiffness in female mice in the setting of WD-induced insulin resistance. Furthermore, we present evidence that DPP-4 inhibition prevented female C57BL/6 J mice from developing WDinduced aorta stiffening, remodeling and dysfunction. We previously reported that WD feeding for 4 months does not increase blood pressure in female C57BL/6J [9]. This previous finding enabled us to design an experiment that 


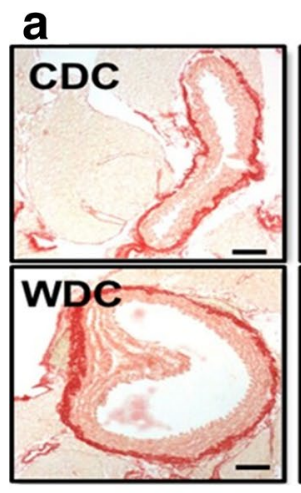

b
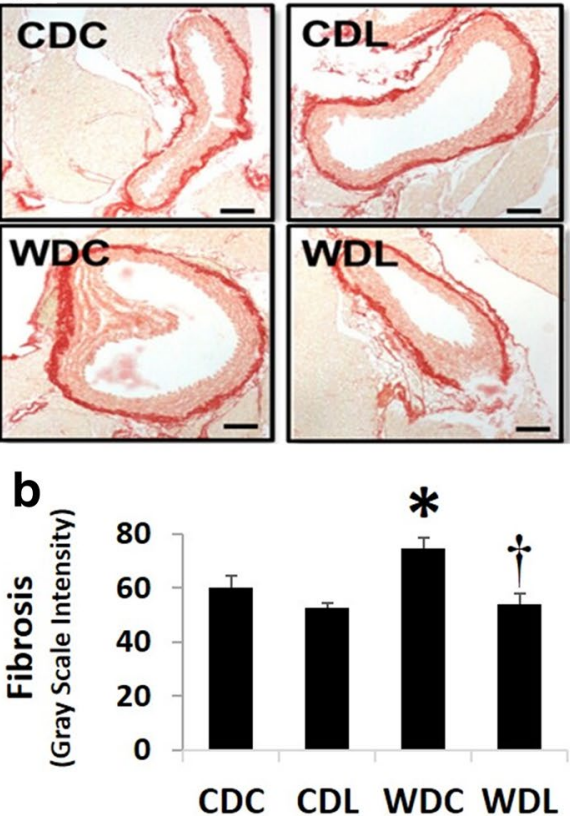

C

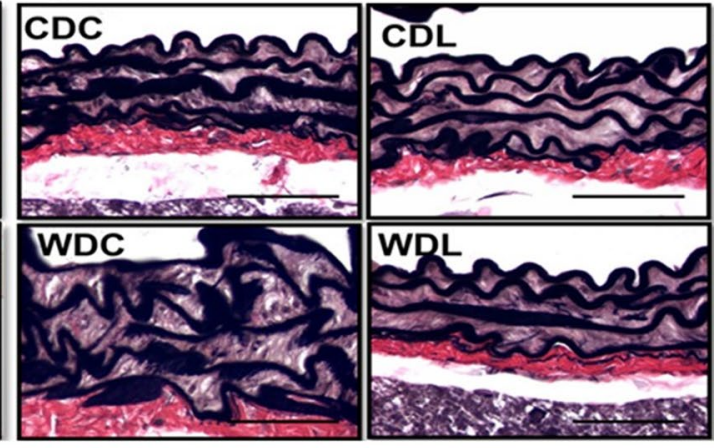

d

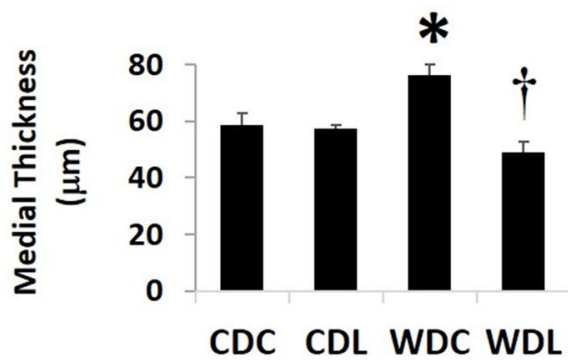

Fig. 4 WD feeding causes $\mathbf{a}$, b peri-aortic fibrosis and (c, d) medial thickening which is ameliorated by the DPP-4 inhibitor, LGT. a Picro sirius red and $\mathbf{b}$ Verhoeff-von Gieson staining. Values are mean \pm SE. CDC control diet control, CDL control diet linagliptin, WDC western diet control and WDL western diet linagliptin. Post-hoc comparisons within a time point; ${ }^{*} p<0.05$ CDC vs WDC; ${ }^{\dagger} p<0.05$ WDC vs WDL

factored out a potential blood pressure effect to explain the CV protective effects of DPP-4 inhibition.

Beneficial vascular effects of DPP-4 inhibition have been attributed to both direct effects of DPP-4 inhibition and to the collateral increase in glucagon like peptide-1 (GLP-1) availability [10]. In the vessel, DPP-4 is located in the cytoplasmic membrane of both EC and vascular smooth muscle cells (VSMC). In EC, DPP-4 expression is increased in models of diabetes [25] and in VSMC, DPP-4 expression is preferentially increased in conditions of vascular remodeling [11]. Importantly, the dose of LGT used in our investigation significantly inhibits DPP-4 activity [12, 14, 26].

Our group previously showed that 4 months of WD feeding in female mice resulted in increases in aortic stiffness, measured non-invasively, as well as EC stiffness, measured ex vivo [9]. Results of this study using a separate cohort of mice were nearly identical with our previous report and further demonstrate aortic and EC stiffening after 4 months of WD feeding. Notably, DPP-4 inhibition prevented the development of aortic stiffness, both in vivo and ex vivo, observed in untreated WD-fed mice. Although previous reports demonstrate that DPP-4 inhibitors decrease cardiac stiffness $[12,27,28]$, to the best of our knowledge this is the first report demonstrating protection from vascular stiffening in a clinically relevant non-diabetic female model of over-nutrition.
Vascular stiffness is determined by EC and VSMC properties, as well as by extracellular matrix and adventitial characteristics [7]. In our experiments, DPP-4 inhibition in the WD-fed cohort prevented the development of impaired endothelial-dependent vasodilation, an effect in the aorta that is known to be largely NOdependent $[29,30]$. In this regard, DPP-4 inhibitors exert direct effects on vascular tone independently of GLP-1 [11]. The DPP-4 inhibitors, LGT and alogliptin, in particular, exert vasodilatory effects in the aorta in the setting of enhanced inflammation and oxidative stress and these effects were mediated by the NO/cGMP pathway [14]. We previously reported a similar improvement in endothelium-dependent vasodilation in skeletal muscle arterioles with LGT administration in a model of obesity and insulin resistance [12]. More recently we showed that impaired NO signaling accompanies increased vascular oxidative stress in our WD-fed mouse model [9]. Thus, it is likely that a decrease in oxidative stress in our WDfed female mice administered a DPP-4 inhibitor, helped to preserve normal aortic vaso-relaxation by preventing NO scavenging by free radicals, such as superoxide, as evidenced by a decrease in 3-nitrotyrosine staining in the aorta compared to untreated mice [7, 31]. In a different model of obesity and insulin resistance, LGT also ameliorated oxidative stress associated with cerebral ischemia independently of glycemic control [32], findings that 

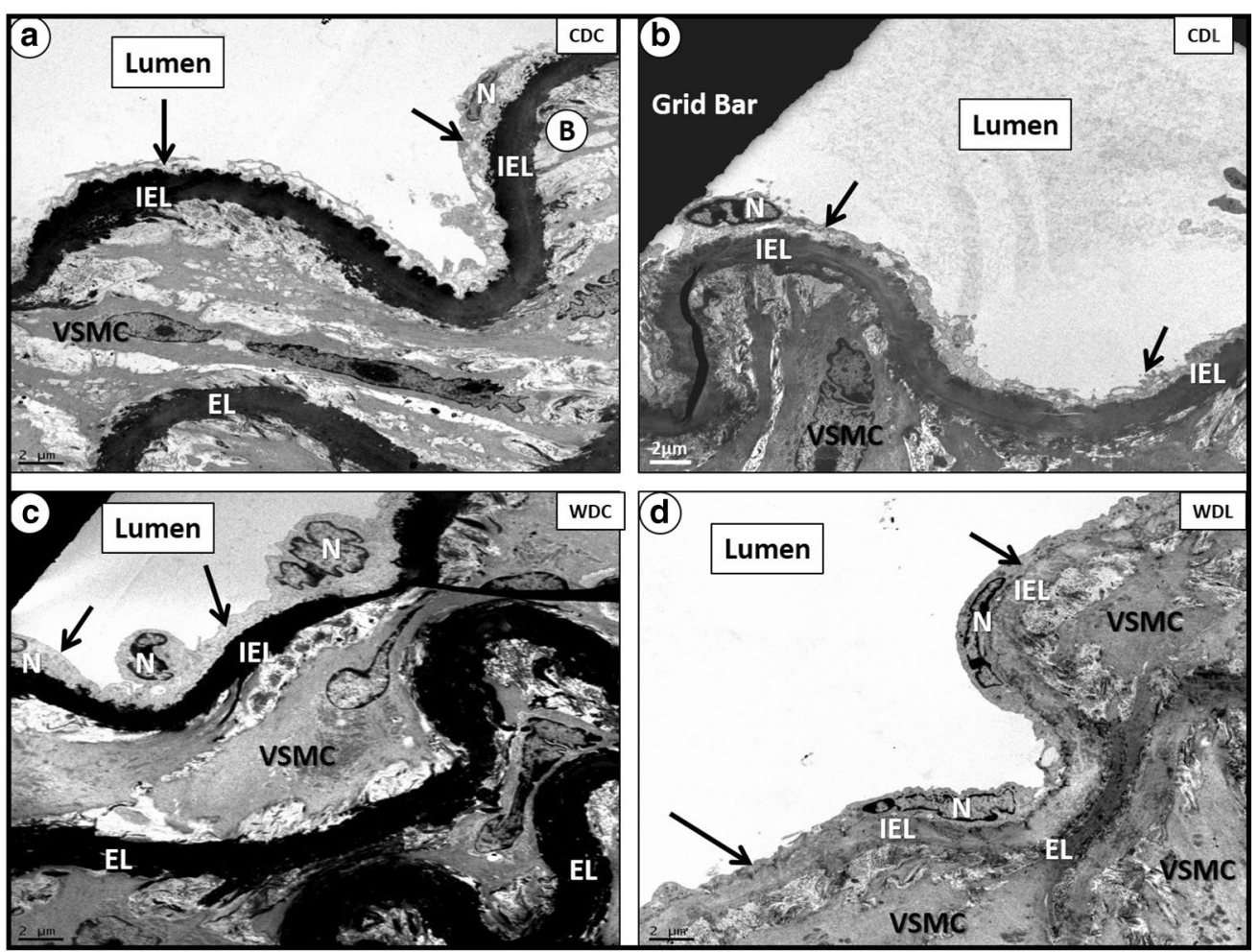

Fig. 5 LGT treatment ameliorates loss of luminal EC cytoplasmic elongation and nuclear contraction induced by WD. a, b depict CDC and CDL cohorts with normal EC characteristics; c depicts contracted cytoplasm and nuclei of EC in WDC; $\mathbf{d}$ demonstrates the absence of luminal EC swelling, edema and contracted cytoplasm and nuclei seen in c. Magnification $\times 800 ;$ bar $2 \mu \mathrm{m}$. EL elastic lamina, IEL internal elastic lamina, $N$ nucleus of luminal endothelial cell, VSMC vascular smooth muscle cell
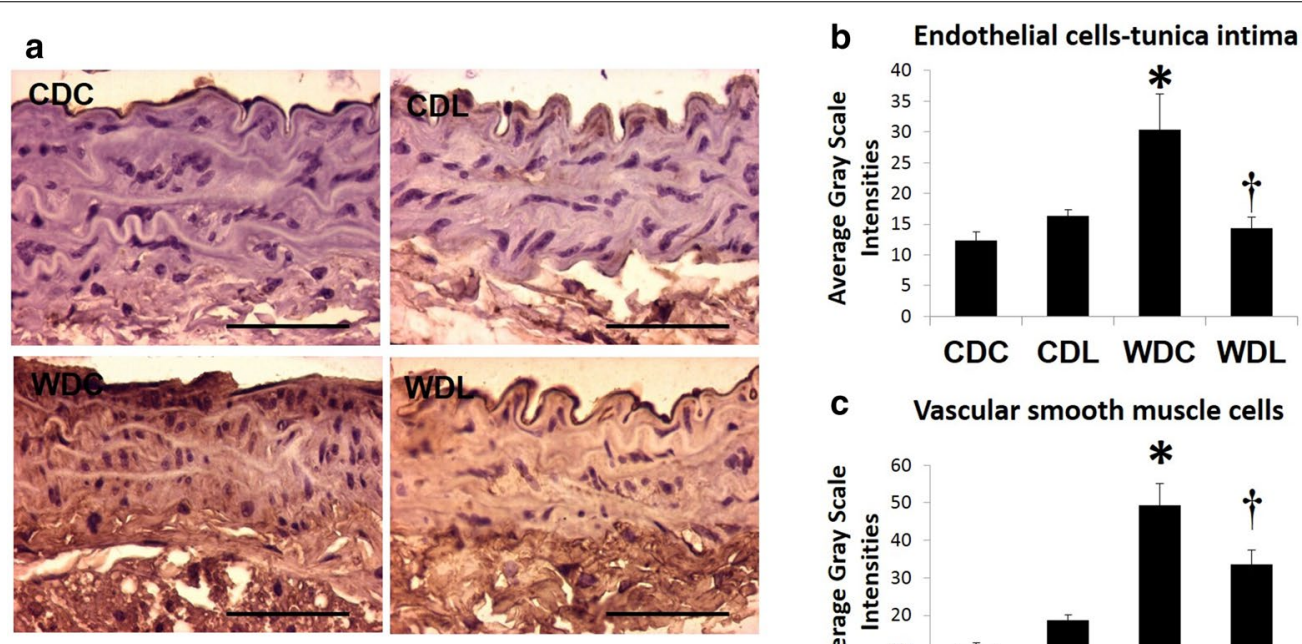

C Vascular smooth muscle cells

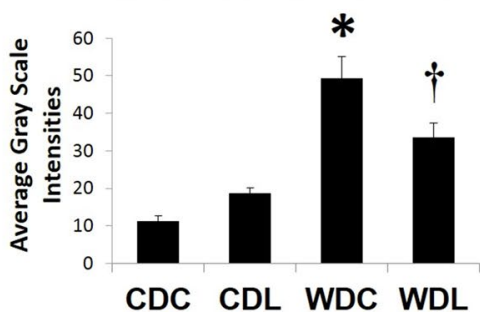

Fig. 6 WD feeding induced aortic oxidative stress is ameliorated with DPP-4 inhibition. a 3-nitrotyrosine staining; b EC 3-nitrotyrosine; c VSMC 3-nitrotyrosine. Values are mean \pm SE. CDC control diet control, CDL control diet linagliptin, WDC western diet control and WDL western diet linagliptin. Post-hoc comparisons within a time point; * $p<00.05$ CDC vs WDC; ${ }^{\dagger} p<0.05$ WDC vs WDL 
a

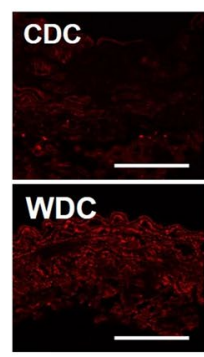

d

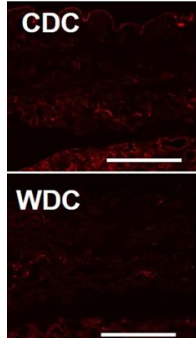

b

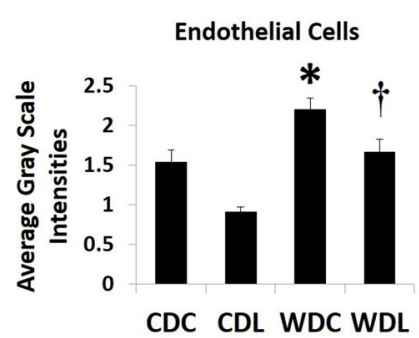

e

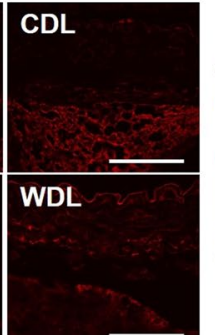

\section{CDL}

WDL

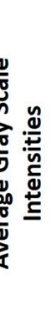

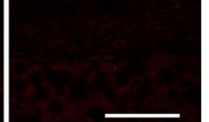

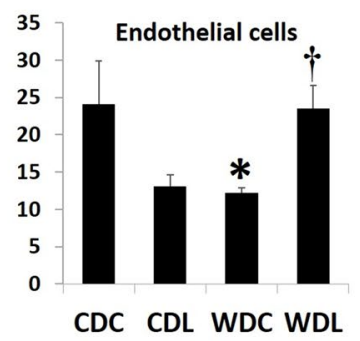

C

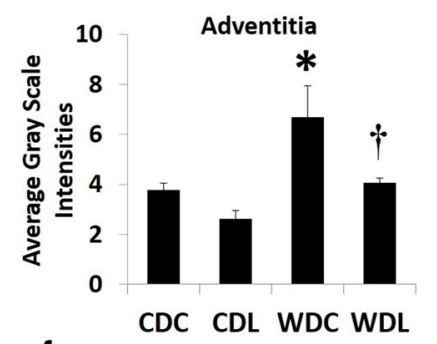

f

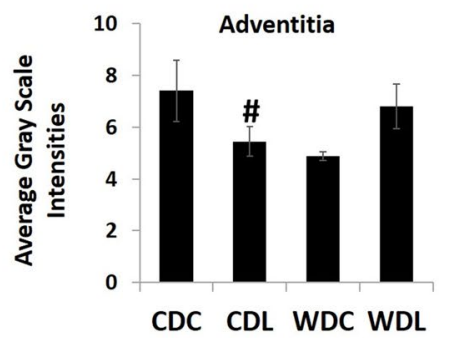

Fig. 7 WD feeding induced changes in FGF-23 and Klotho expression are restored by DPP-4 inhibition. a FGF-23 staining; b Endothelial FGF-23; c Adventitia FGF-23; d Endothelial klotho staining; e Endothelial klotho staining $\mathbf{f}$ Adventitia klotho staining; Average gray intensities in the different cohorts. Values are mean \pm SE. CDC control diet control, CDL control diet linagliptin, WDC western diet control and WDL western diet linagliptin. Post-hoc comparisons within a time point; ${ }^{*} p<00.05$ CDC vs WDC; ${ }^{\dagger} p<0.05$ WDC vs WDL; ${ }^{p} p<0.05$ CDC vs CDL. Scale bars represent $50 \mathrm{~m} \mu$

further support the concept that the antioxidant properties of LGT may be vasculoprotective.

Likewise, the beneficial role of DPP-4 inhibition in restoring endothelial-dependent vasodilation in conditions of increased oxidative stress has been documented in other murine models [33,34]. It should be noted that even though the magnitude of the aortic vasodilatory responses reported in this investigation seem low, similar results in mice have been reported from other laboratories [35-37]. Furthermore, our ultra-structural findings suggest that DPP-4 inhibition reverses the loss of luminal endothelial cytoplasmic elongations and nuclear contraction associated with WD feeding. Additionally, our findings indicate that the beneficial effects of the DPP-4 inhibition are not limited to the endothelium. Here, we observed that DPP-4 inhibition decreases medial thickening and fibrosis related to WD-feeding. The vascular anti-fibrotic role of DPP-4 inhibitors has been reported previously in models of atherosclerosis and neo-intima injury $[34,38]$.

FGF-23 is elevated in conditions of insulin resistance and obesity $[39,40]$. Importantly, FGF-23 levels are associated with impaired vascular relaxation, vascular calcification and stiffness [22, 40, 41]. Moreover, FGF-23 is expressed in the vasculature [42] and has been shown to enhance oxidative stress and induce inhibition of NOdependent vasodilation [43]. We recently reported that female WD-fed mice exhibit increased FGF-23 levels and this was associated with impaired aortic endothelial function, increased aortic PWV and increased oxidative stress [24]. FGF-23 effects may be modulated, in part, via Klotho [44]. In this regard, Klotho deficiency was recently reported to accelerate aortic stiffening in high fat fed mice [45]. Whether WD leads to a deficiency in Klotho expression has not been examined previously. Herein, we explored a possible contribution of FGF-23 and Klotho to the genesis of vascular stiffening, endothelial dysfunction and oxidative stress by testing whether DPP-4 inhibition prevents the increase in aortic FGF-23 expression and modulates Klotho expression in the aorta of WDfed mouse model. In the present work, FGF-23 expression was increased in the aorta of WD-fed mice and this was prevented with DPP-4 inhibition. Furthermore, the expression of Klotho was decreased in the aorta of WDfed mice relative to control mice and this deficiency was prevented with administration of LGT. In this regard and relevant to our findings, others have reported that Klotho deficiency results in increased production of reactive oxygen [46], while its over-expression ameliorates oxidative stress [47]. Since oxidative stress has been implicated in the impairment of vascular relaxation and bioavailable NO, the prevention of abnormal expression of FGF23 and Klotho by DPP-4 inhibition in the vasculature may represent a new mechanism to explain the efficacy of DPP-4 inhibition in preventing the appearance of vascular stiffness. Whether FGF-23/Klotho signaling in the 
vasculature, in concert with its vascular receptors and cofactors, directly modulates vascular function or influences vascular function via regulation of putative effects on mineral metabolism is unclear.

In the present investigation we did not observe that LGT prevented or reduced WD-induced weight gain suggesting that the efficacy of LGT in preventing WDinduced aortic stiffening, impaired vasoreactivity and abnormal remodeling did not result from beneficial weight loss. Some rodent studies have documented a similar neutral effect of LGT administration on body weight in association with improvement in cardiovascular function and structure $[25,48]$. On the other hand, higher doses of LGT led to modest weight loss in rats fed a high fat diet [49]. Importantly clinical trials have supported the notion of weight stability with LGT treatment [50-52]. One of the limitations of the present investigation is that we did not directly evaluate glucose homeostasis or insulin resistance. Nevertheless, we have previously demonstrated that this diet paradigm in female C57BL/6J mice does not result in frank hyperglycemia while it manifests significant whole body insulin resistance evaluated by the hyperinsulinemic euglycemic clamp [53]. Herein, we further explored the possibility that DPP-4 inhibition reduces the anticipated increase in expression of AGE products as AGE/RAGE signaling can result in a pro-inflammatory and pro-oxidant environment [54]. Furthermore, AGE can increase expression of DPP-4 in EC [55]. In the present investigation, we documented increased AGE presence in the vascular wall of WD-fed females, and this was not prevented with DPP-4 inhibition. This suggests that the efficacy of DPP-4 inhibition in the vasculature of WD-fed female mice occurs without significant changes in AGE. To the best of our knowledge this is the only study demonstrating that a DPP-4 inhibitor prevents development of diet-induced vascular stiffness. Further studies are needed to clarify if other DPP-4 inhibitors share these beneficial effects.

Despite abundant preclinical evidence of cardiovascular benefits of DPP-4 inhibitors, including results presented herein, meta-analyses of clinical data raise concerns regarding evidence of increased risk of heart failure with administration of certain DPP-4 inhibitors [56]. On the other hand, other recent analyses have produced differing conclusions regarding heart failure risk assessment [57]. In the SAVOR-TIMI 53 trial the use of saxagliptin was associated with increased risk of heart failure [58]. Importantly, the risk was higher in the subjects with previous heart failure, elevated natriuretic peptides and impaired renal function [59]. In the EXAMINE trial that explored the use of alogliptin in type 2 diabetics with post-acute coronary events there was no increased risk of major CV events [60]. Nevertheless, a recent post hoc analysis of the EXAMINE trial did find a statistically significant increase in hospitalization rate from heart failure in the group of subjects without heart failure history [61]. On the contrary, the sitagliptin TECOS trial did not find differences in the rate of hospitalization for heart failure between the treatment and the control group [62]. Similarly, a recent pre-specified patient-level pooled analysis of available trials of LGT did not report an association between the DPP-4 inhibitor and increased CV risk (including heart failure) [63]. Results from two ongoing trials using LGT, the CAROLINA (NCT01243424) and CARMELINA (NCT01897532), will further clarify the role and safety of this agent in regard to diabetic CVD.

To summarize, the current investigation demonstrates that DPP-4 inhibition prevents abnormal increases in vascular stiffness, aortic fibrosis, oxidative stress and FGF-23/Klotho expression induced by a WD in female mice and these benefits occur independent of AGE reduction. Our findings have clinical relevance as obese diabetic women are more frequently affected by increased vascular stiffness that likely promotes higher incident CVD compared to their male counterparts. Ultimately, only the results of well-designed clinical trials will clarify the role of LGT in the management of diabetic CVD.

\section{Additional file}

Additional file 1: Table S1. Body weights of C57BL/6J mice fed a western diet (WD) high in fat and high fructose corn syrup compared to mice fed a control diet (CD). WD induced significant increases in body weight in WDC and WDL compared to their respective control groups. Administration of linagliptin $(L)$ had no effect on body weight.

\section{Abbreviations \\ DPP-4: dipeptidylpeptidase-4; WD: western diet; LGT: linagliptin; PWV: pulse wave velocity; AFM: atomic force microscopy; CD: control diet; CV: cardiovas- cular; CDC: control diet without LGT; CDL: control diet with LGT; WDC: western diet without LGT; WDL: western diet with LGT; Akt: protein kinase B; eNOS: endothelial nitric oxide synthase; Ach: acetylcholine.}

\section{Authors' contributions}

VGD, AA, TK and JRS made substantial contributions to conception and study design. CM, VGD and AA were involved in drafting and revising the manuscript, including statistical analysis and data interpretation, and graphics. JH, AA, GJ, MRH, MG, LAM, FIR, GAM, JRS and VGD contributed to the acquisition and interpretation of data and associated intellectual content. All authors read and approved the final manuscript.

\footnotetext{
Author details

${ }^{1}$ Department of Internal Medicine, Division of Endocrinology, Diabetes and Metabolism, University of Missouri-Columbia School of Medicine, One Hospital Drive, Columbia, MO 65212, USA. ${ }^{2}$ MU Diabetes and Cardiovascular Research Center, University of Missouri, School of Medicine, Columbia, USA. ${ }^{3}$ Department of Medical Pharmacology and Physiology, University of Missouri, School of Medicine, Columbia, USA. ${ }^{4}$ Research Service, Harry S. Truman Memorial Veterans Hospital, Columbia, USA. ${ }^{5}$ The Dalton Cardiovascular Research Center, Columbia, USA. ${ }^{6}$ Boehringer Ingelheim Pharma, Ingelheim Am Rhein, Germany.
} 


\begin{abstract}
Acknowledgements
We gratefully acknowledge Brenda Hunter for editorial assistance, Nathan Rehmer, Dongqing Chen, and Brady Barron for technical support. This work was supported with resources and facilities at the University of Missouri, the Harry S. Truman Memorial Veterans Hospital in Columbia, MO, including the Small Animal Ultrasound Imaging Center (Echocardiography), as well as the Atomic Force Microscopy Center in the Dalton Cardiovascular Research Center.
\end{abstract}

\section{Competing interests}

VGD received support from Boehringer Ingelheim, TK is an employee of Boehringer Ingelheim.

\section{Availability of data and materials}

Not applicable. The conclusions of the manuscript are based on relevant data sets available in the manuscript.

\section{Sources of funding}

The design of the study and collection, analysis, and interpretation of data and writing of this manuscript was supported by an unrestricted research Grant from Boehringer Ingelheim Pharma (VGD), as well as support from the National Institutes of Health (1 K08HL129074-01 to CM, R01-HL073101 RO1-HL107910 to JRS, R01 HL088105 LAM-L and P01 HL095486 to GAM), Department of Veterans Affairs Merit Award 1BX001981 to JRS, and Boehringer Ingelheim Pharma to TK.

Received: 5 April 2016 Accepted: 23 June 2016

Published online: 08 July 2016

\section{References}

1. Coutinho T. Arterial stiffness and its clinical implications in women. Can J Cardiol. 2014;30:756-64.

2. Weisbrod RM, Shiang T, Alsayah L, Fry JL, Bajpai S, Reinhartking CA, Lob HE, Santhanam L, Mitchell G, Cohen RA, Seta F. Arterial stiffening precedes systolic hypertension in diet-induced obesity. Hypertension. 2013;62:1105-10

3. Webb DR, Khunti K, Silverman R, Gray LJ, Srinivasan B, Lacy PS, Williams B, Davies MJ. Impact of metabolic indices on central artery stiffness: independent association of insulin resistance and glucose with aortic pulse wave velocity. Diabetologia. 2010;53:1190-8.

4. Park JS, Nam JS, Cho MH, Yoo JS, Ahn CW, Jee SH, Lee HS, Cha BS, Kim $\mathrm{KR}$, Lee HC. Insulin resistance independently influences arterial stiffness in normoglycemic normotensive postmenopausal women. Menopause (NY). 2010;17:779-84.

5. Malik VS, Popkin BM, Bray GA, Despres JP, Willett WC, Hu FB. Sugar-sweetened beverages and risk of metabolic syndrome and type 2 diabetes: a meta-analysis. Diabetes Care. 2010;33:2477-83.

6. Malik VS, Hu FB. Fructose and cardiometabolic health: what the evidence from sugar-sweetened beverages tells us. J Am Coll Cardiol. 2015;66:1615-24

7. Aroor AR, Demarco VG, Jia G, Sun Z, Nistala R, Meininger GA, Sowers JR. The role of tissue Renin-Angiotensin-aldosterone system in the development of endothelial dysfunction and arterial stiffness. Front Endocrinol. 2013;4:161.

8. Leopold JA. Cellular and molecular mechanisms of arterial stiffness associated with obesity. Hypertension. 2013;62:1003-4.

9. DeMarco VG, Habibi J, Jia G, Aroor AR, Ramirez-Perez Fl, Martinez-Lemus LA, Bender SB, Garro M, Hayden MR, Sun Z, Meininger GA, Manrique C, Whaley-Connell A, Sowers JR. Low-dose mineralocorticoid receptor blockade prevents western diet-induced arterial stiffening in female mice. Hypertension. 2015;66:99-107.

10. Jose T, Inzucchi SE. Cardiovascular effects of the DPP-4 inhibitors. Diabetes Vasc Dis Res. 2012;9:109-16.

11. Aroor AR, Sowers JR, Jia G, DeMarco VG. Pleiotropic effects of the dipeptidylpeptidase-4 inhibitors on the cardiovascular system. Am J Physiol Heart Circ Physiol. 2014;307:H477-92.

12. Aroor AR, Sowers JR, Bender SB, Nistala R, Garro M, Mugerfeld I, Hayden MR, Johnson MS, Salam M, Whaley-Connell A, Demarco VG. Dipeptidylpeptidase inhibition is associated with improvement in blood pressure and diastolic function in insulin-resistant male Zucker obese rats. Endocrinology. 2013;154:2501-13.

13. Eckhardt M, Langkopf E, Mark M, Tadayyon M, Thomas L, Nar H, Pfrengle W, Guth B, Lotz R, Sieger P, Fuchs H, Himmelsbach F. 8-(3-(R)-aminopiperidin-1-yl)-7-but-2-ynyl-3-methyl-1-(4-methyl-quinazolin-2-ylme thyl)-3,7-dihydropurine-2,6-dione (BI 1356), a highly potent, selective, long-acting, and orally bioavailable DPP-4 inhibitor for the treatment of type 2 diabetes. J Med Chem. 2007;50:6450-3.

14. Kroller-Schon S, Knorr M, Hausding M, Oelze M, Schuff A, Schell R, Sudowe S, Scholz A, Daub S, Karbach S, Kossmann S, Gori T, Wenzel P, Schulz E, Grabbe S, Klein T, Munzel T, Daiber A. Glucose-independent improvement of vascular dysfunction in experimental sepsis by dipeptidyl-peptidase 4 inhibition. Cardiovasc Res. 2012;96:140-9.

15. Nistala R, Habibi J, Lastra G, Manrique C, Aroor AR, Hayden MR, Garro M, Meuth A, Johnson M, Whaley-Connell A, Sowers JR. Prevention of obesityinduced renal injury in male mice by DPP4 inhibition. Endocrinology. 2014;155:2266-76.

16. Zhou X, Ma L, Habibi J, Whaley-Connel AT, Hayden MR, Tilmon RD, Brown AN, DeMarco VG, Sowers JR. Nebivolol improves diastolic dysfunction and myocardial tissue remodeling through reductions in oxidative stress in the Zucker Obese rat. Hypertension. 2010;55:880-8.

17. Wei Y, Whaley-Connell AT, Habibi J, Rehmer J, Rehmer N, Patel K, Hayden M, DeMarco V, Ferrario CM, Ibdah JA, Sowers JR. Mineralocorticoid receptor antagonism attenuates vascular apoptosis and injury via rescuing protein kinase B activation. Hypertension. 2009;53:158-65.

18. Llaurado G, Ceperuelo-Mallafre V, Vilardell C, Simo R, Gil P, Cano A, Vendrell J, Gonzalez-Clemente JM. Advanced glycation end products are associated with arterial stiffness in type 1 diabetes. J Endocrinol. 2014:221:405-13.

19. Goldin A, Beckman JA, Schmidt AM, Creager MA. Advanced glycation end products: sparking the development of diabetic vascular injury. Circulation. 2006;114:597-605.

20. Huveneers S, Daemen MJ, Hordijk PL. Between Rho(k) and a hard place: the relation between vessel wall stiffness, endothelial contractility, and cardiovascular disease. Circ Res. 2015;116:895-908.

21. Kestenbaum B, Sachs MC, Hoofnagle AN, Siscovick DS, Ix JH, RobinsonCohen C, Lima JA, Polak JF, Blondon M, Ruzinski J, Rock D, de Boer IH. Fibroblast growth factor-23 and cardiovascular disease in the general population: the multi-ethnic study of atherosclerosis. Circ Heart Fail. 2014;7:409-17.

22. di Giuseppe R, Kuhn T, Hirche F, Buijsse B, Dierkes J, Fritsche A, Kaaks R, Boeing $\mathrm{H}$, Stangl Gl, Weikert C. Potential predictors of plasma fibroblast growth factor 23 concentrations: cross-sectional analysis in the EPICgermany study. PLOS ONE. 2015;10:e0133580.

23. Lutsey PL, Alonso A, Selvin E, Pankow JS, Michos ED, Agarwal SK, Loehr LR, Eckfeldt $J \mathrm{H}$, Coresh J. Fibroblast growth factor-23 and incident coronary heart disease, heart failure, and cardiovascular mortality: the atherosclerosis risk in communities study. J Am Heart Assoc. 2014;3:e000936.

24. Jia G, Habibi J, Aroor AR, Martinezlemus LA, DeMarco VG, Ramirez-Perez FI, Sun Z, Hayden MR, Meininger GA, Barrettmueller KV, Jaffe IZ, Sowers $J R$. Endothelial mineralocorticoid receptor mediates diet induced aortic stiffness in females. Circ Res. 2016;118:935.

25. Kanasaki K, Shi S, Kanasaki M, He J, Nagai T, Nakamura Y, Ishigaki Y, Kitada M, Srivastava SP, Koya D. Linagliptin-mediated DPP-4 inhibition ameliorates kidney fibrosis in streptozotocin-induced diabetic mice by inhibiting endothelial-to-mesenchymal transition in a therapeutic regimen. Diabetes. 2014;63:2120-31.

26. Sharkovska Y, Reichetzeder C, Alter M, Tsuprykov O, Bachmann S, Secher T, Klein T, Hocher B. Blood pressure and glucose independent renoprotective effects of dipeptidyl peptidase-4 inhibition in a mouse model of type-2 diabetic nephropathy. J Hypertension. 2014;32:2211-23.

27. Hamdani N, Hervent AS, Vandekerckhove L, Matheeussen V, Demolder M, Baerts L, De Meester I, Linke WA, Paulus WJ, De Keulenaer GW. Left ventricular diastolic dysfunction and myocardial stiffness in diabetic mice is attenuated by inhibition of dipeptidyl peptidase 4. Cardiovasc Res. 2014;104:423-31 (discussion 2223-2227).

28. Bostick B, Habibi J, Ma L, Aroor A, Rehmer N, Hayden MR, Sowers JR. Dipeptidyl peptidase inhibition prevents diastolic dysfunction and reduces myocardial fibrosis in a mouse model of Western diet induced obesity. Metab Clin Exp. 2014;63:1000-11. 
29. Vanhoutte PM. Endothelium and control of vascular function. State of the Art lecture. Hypertension. 1989;13:658-67.

30. Chataigneau T, Feletou M, Huang PL, Fishman MC, Duhault J, Vanhoutte PM. Acetylcholine-induced relaxation in blood vessels from endothelial nitric oxide synthase knockout mice. Br J Pharmacol. 1999;126:219-26.

31. DeMarco VG, Aroor AR, Sowers JR. The pathophysiology of hypertension in patients with obesity. Nat Rev Endocrinol. 2014;10:364-76.

32. Ma M, Hasegawa Y, Koibuchi N, Toyama K, Uekawa K, Nakagawa T, Lin B, Kim-Mitsuyama S. DPP-4 inhibition with linagliptin ameliorates cognitive impairment and brain atrophy induced by transient cerebral ischemia in type 2 diabetic mice. Cardiovasc Diabetol. 2015;14:54.

33. Salim HM, Fukuda D, Higashikuni Y, Tanaka K, Hirata Y, Yagi S, Soeki T, Shimabukuro M, Sata M. Dipeptidyl peptidase-4 inhibitor, linagliptin, ameliorates endothelial dysfunction and atherogenesis in normoglycemic apolipoprotein-E deficient mice. Vasc Pharmacol. 2015;79:16.

34. Zeng Y, Li C, Guan M, Zheng Z, Li J, Xu W, Wang L, He F, Xue Y. The DPP-4 inhibitor sitagliptin attenuates the progress of atherosclerosis in apolipoprotein-E-knockout mice via AMPK- and MAPK-dependent mechanisms. Cardiovasc Diabetol. 2014;13:32.

35. Pojoga LH, Yao TM, Sinha S, Ross RL, Lin JC, Raffetto JD, Adler GK, Williams $\mathrm{GH}$, Khalil RA. Effect of dietary sodium on vasoconstriction and eNOSmediated vascular relaxation in caveolin-1-deficient mice. Am J Physiol Heart Circ Physiol. 2008;294:H1258-65.

36. Pojoga LH, Adamova Z, Kumar A, Stennett AK, Romero JR, Adler GK, Williams GH, Khalil RA. Sensitivity of NOS-dependent vascular relaxation pathway to mineralocorticoid receptor blockade in caveolin-1-deficient mice. Am J Physiol Heart Circ Physiol. 2010;298:H1776-88.

37. Pojoga LH, Yao TM, Opsasnick LA, Garza AE, Reslan OM, Adler GK, Williams GH, Khalil RA. Dissociation of hyperglycemia from altered vascular contraction and relaxation mechanisms in caveolin-1 null mice. J Pharmacol Exp Ther. 2014;348:260-70.

38. Terawaki Y, Nomiyama T, Kawanami T, Hamaguchi Y, Takahashi H, Tanaka T, Murase K, Nagaishi R, Tanabe M, Yanase T. Dipeptidyl peptidase-4 inhibitor linagliptin attenuates neointima formation after vascular injury. Cardiovasc Diabetol. 2014;13:154.

39. Mirza MA, Alsio J, Hammarstedt A, Erben RG, Michaelsson K, Tivesten A, Marsell R, Orwoll E, Karlsson MK, Ljunggren O, Mellstrom D, Lind L, Ohlsson C, Larsson TE. Circulating fibroblast growth factor-23 is associated with fat mass and dyslipidemia in two independent cohorts of elderly individuals. Arterioscler Thromb Vasc Biol. 2011;31:219-27.

40. Hanks $\sqcup$, Casazza K, Judd SE, Jenny NS, Gutierrez OM. Associations of fibroblast growth factor-23 with markers of inflammation, insulin resistance and obesity in adults. PLoS ONE. 2015;10:e122885.

41. Tsao CW, Pencina KM, Massaro JM, Benjamin EJ, Levy D, Vasan RS, Hoffmann U, O'Donnell CJ, Mitchell GF. Cross-sectional relations of arterial stiffness, pressure pulsatility, wave reflection, and arterial calcification. Arterioscler Thromb Vasc Biol. 2014;34:2495-500.

42. Fang $Y$, Ginsberg C, Seifert M, Agapova O, Sugatani T, Register TC, Freedman BI, Monier-Faugere MC, Malluche H, Hruska KA. CKD-induced wingless/integration 1 inhibitors and phosphorus cause the CKD-mineral and bone disorder. J Am Soc Nephrol. 2014;25:1760-73.

43. Silswal N, Touchberry CD, Daniel DR, McCarthy DL, Zhang S, Andresen J, Stubbs JR, Wacker MJ. FGF23 directly impairs endothelium-dependent vasorelaxation by increasing superoxide levels and reducing nitric oxide bioavailability. Am J Physiol Endocrinol Metab. 2014;307:E426-36.

44. Urakawa I, Yamazaki Y, Shimada T, lijima K, Hasegawa H, Okawa K, Fujita T, Fukumoto S, Yamashita T. Klotho converts canonical FGF receptor into a specific receptor for FGF23. Nature. 2006;444:770-4.

45. Lin Y, Chen J, Sun Z. Antiaging gene Klotho deficiency promoted high-fat diet-induced arterial stiffening via inactivation of AMP-activated protein kinase. Hypertension. 2016;67:564

46. Izbeki F, Asuzu DT, Lorincz A, Bardsley MR, Popko LN, Choi KM, Young DL, Hayashi Y, Linden DR, Kuro-o M, Farrugia G, Ordog T. Loss of Kitlow progenitors, reduced stem cell factor and high oxidative stress underlie gastric dysfunction in progeric mice. J Physiol. 2010;588:3101-17.

47. Wang Y, Kuro-o M, Sun Z. Klotho gene delivery suppresses Nox2 expression and attenuates oxidative stress in rat aortic smooth muscle cells via the cAMP-PKA pathway. Aging Cell. 2012;11:410-7.

48. Salim HM, Fukuda D, Higashikuni Y, Tanaka K, Hirata Y, Yagi S, Soeki T, Shimabukuro M, Sata M. Dipeptidyl peptidase-4 inhibitor, linagliptin, ameliorates endothelial dysfunction and atherogenesis in normoglycemic apolipoprotein-E deficient mice. Vascul Pharmacol. 2016;79:16-23.

49. Hansen HH, Hansen G, Paulsen S, Vrang N, Mark M, Jelsing J, Klein T. The DPP-IV inhibitor linagliptin and GLP-1 induce synergistic effects on body weight loss and appetite suppression in the diet-induced obese rat. Eur J Pharmacol. 2014;741:254-63.

50. Barnett AH, Huisman H, Jones R, von Eynatten M, Patel S, Woerle HJ. Linagliptin for patients aged 70 years or older with type 2 diabetes inadequately controlled with common antidiabetes treatments: a randomised, double-blind, placebo-controlled trial. Lancet. 2013;382:1413-23.

51. Yki-Jarvinen H, Rosenstock J, Duran-Garcia S, Pinnetti S, Bhattacharya S, Thiemann S, Patel S, Woerle HJ. Effects of adding linagliptin to basal insulin regimen for inadequately controlled type 2 diabetes: $\mathrm{a}>/=52$-week randomized, double-blind study. Diabetes Care. 2013;36:3875-81.

52. Gallwitz B, Rosenstock J, Rauch T, Bhattacharya S, Patel S, von Eynatten M, Dugi KA, Woerle HJ. 2-year efficacy and safety of linagliptin compared with glimepiride in patients with type 2 diabetes inadequately controlled on metformin: a randomised, double-blind, non-inferiority trial. Lancet. 2012;380:475-83.

53. Manrique C, Demarco VG, Aroor AR, Mugerfeld I, Garro M, Habibi J, Hayden MR, Sowers JR. Obesity and insulin resistance induce early development of diastolic dysfunction in young female mice fed a western diet. Endocrinology. 2013;154:3632-42.

54. Yan SF, Ramasamy R, Schmidt AM. The RAGE axis: a fundamental mechanism signaling danger to the vulnerable vasculature. Circ Res. 2010;106:842-53.

55. Ishibashi Y, Matsui T, Maeda S, Higashimoto Y, Yamagishi S. Advanced glycation end products evoke endothelial cell damage by stimulating soluble dipeptidyl peptidase-4 production and its interaction with mannose 6-phosphate/insulin-like growth factor II receptor. Cardiovasc Diabetol. 2013:12:125.

56. Li L, Li S, Deng K, Liu J, Vandvik PO, Zhao P, Zhang L, Shen J, Bala MM, Sohani ZN, Wong E, Busse JW, Ebrahim S, Malaga G, Rios LP, Wang Y, Chen Q, Guyatt GH, Sun X. Dipeptidyl peptidase-4 inhibitors and risk of heart failure in type 2 diabetes: systematic review and meta-analysis of randomised and observational studies. BMJ. 2016;352:610.

57. Filion KB, Azoulay L, Platt RW, Dahl M, Dormuth CR, Clemens KK, Hu N, Paterson JM, Targownik L, Turin TC, Udell JA, Ernst P, Investigators C. A multicenter observational study of incretin-based drugs and heart failure. N Engl J Med. 2016;374:1145-54.

58. Scirica BM, Bhatt DL, Braunwald E, Steg PG, Davidson J, Hirshberg B, Ohman P, Frederich R, Wiviott SD, Hoffman EB, Cavender MA, Udell JA, Desai NR, Mosenzon O, McGuire DK, Ray KK, Leiter LA, Raz I. Saxagliptin and cardiovascular outcomes in patients with type 2 diabetes mellitus. New Engl J Med. 2013;369:1317-26.

59. Scirica BM, Braunwald E, Raz I, Cavender MA, Morrow DA, Jarolim P, Udell JA, Mosenzon O, Im K, Umez-Eronini AA, Pollack PS, Hirshberg B, Frederich R, Lewis BS, McGuire DK, Davidson J, Steg PG, Bhatt DL. Heart failure, saxagliptin, and diabetes mellitus: observations from the SAVOR-TIMI 53 randomized trial. Circulation. 2014;130:1579-88.

60. White WB, Cannon CP, Heller SR, Nissen SE, Bergenstal RM, Bakris GL, Perez AT, Fleck PR, Mehta CR, Kupfer S, Wilson C, Cushman WC, Zannad F. Alogliptin after acute coronary syndrome in patients with type 2 diabetes. New Engl J Med. 2013;369:1327-35.

61. Zannad F, Cannon CP, Cushman WC, Bakris GL, Menon V, Perez AT, Fleck PR, Mehta CR, Kupfer S, Wilson C, Lam H, White WB, Investigators E. Heart failure and mortality outcomes in patients with type 2 diabetes taking alogliptin versus placebo in EXAMINE: a multicentre, randomised, double-blind trial. Lancet. 2015;385:2067-76.

62. Green JB, Bethel MA, Armstrong PW, Buse JB, Engel SS, Garg J, Josse R, Kaufman KD, Koglin J, Korn S, Lachin JM, McGuire DK, Pencina MJ, Standl E, Stein PP, Suryawanshi S, Vande Werf F, Peterson ED, Holman RR, Group TS. Effect of sitagliptin on cardiovascular outcomes in type 2 diabetes. N Engl J Med. 2015;373:232-42.

63. Rosenstock J, Marx N, Neubacher D, Seck T, Patel S, Woerle HJ, Johansen OE. Cardiovascular safety of linagliptin in type 2 diabetes: a comprehensive patient-level pooled analysis of prospectively adjudicated cardiovascular events. Cardiovasc Diabetol. 2015;14:57. 